\title{
Production of Butyrate and Branched Chain Amino Acid Catabolic Byproducts by CHO Cells in Fed-batch Culture Enhances their Specific Productivity
}

\author{
Cameron Harrington ${ }^{1}$, Taylor Kalomeris ${ }^{2}$, Michaela Jacobs² ${ }^{2}$ Gregory Hiller ${ }^{3}$, and Bhanu \\ Chandra Mulukutla ${ }^{2}$ \\ ${ }^{1}$ Pfizer Andover \\ ${ }^{2}$ Pfizer \\ ${ }^{3}$ Pfizer, Inc.
}

February 11, 2021

\begin{abstract}
Chinese hamster ovary $(\mathrm{CHO})$ cells in fed-batch cultures produce several metabolic byproducts derived from amino acid catabolism, some of which accumulate to growth inhibitory levels. Controlling the accumulation of these byproducts has been shown to significantly enhance cell proliferation. Interestingly, some of these byproducts have physiological roles that go beyond inhibition of cell proliferation. In this study, we show that, in CHO cell fed-batch cultures, branched chain amino acid (BCAA) catabolism contributes to the formation of butyrate, a novel byproduct that is also a well-established specific productivity enhancer. Further, the other byproducts of BCAA catabolism, isovalerate and isobutyrate, which accumulate in CHO cell fed-batch cultures also enhance specific productivity. Additionally, the rate of production of these BCAA catabolic byproducts was negatively correlated with glucose uptake and lactate production rates. Limiting glucose supply to suppress glucose uptake and lactate production, like in case of fed-batch cultures employing HiPDOG technology, significantly enhances BCAA catabolic byproduct accumulation resulting in higher specific productivities.
\end{abstract}

\section{Hosted file}

Main Manuscript File.pdf available at https://authorea.com/users/390259/articles/508402production-of-butyrate-and-branched-chain-amino-acid-catabolic-byproducts-by-cho-cellsin-fed-batch-culture-enhances-their-specific-productivity 
Figure 1: SCFA and butyrate levels in multiple $\mathrm{CHO}$ cell line cultures with low amino acid process or with BCAT1 knocked out.
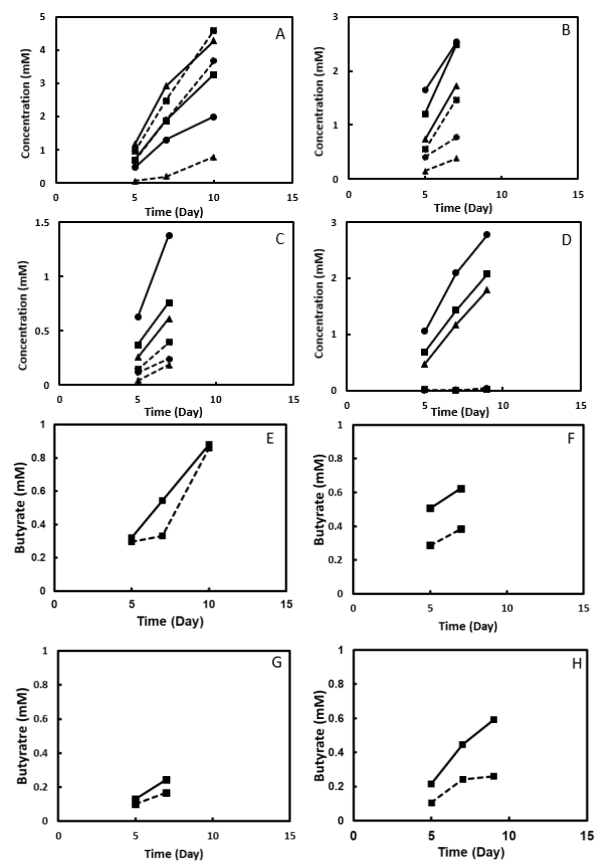
Figure 11: Day 7 Specific butyrate production rate of cell line $C$ across conditions with limited residual culture levels of one or more branched chain amino acids.

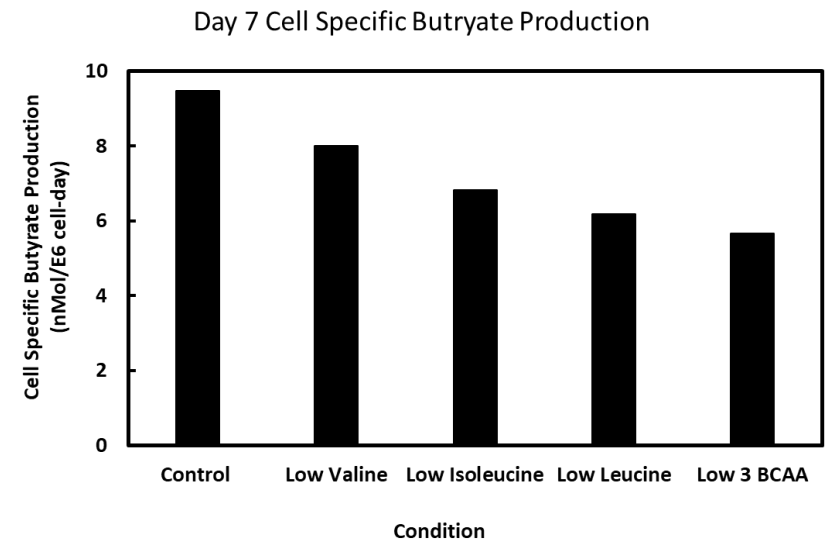


Figure 2: $q_{P}$ enhancing effect of butyrate and isovalerate on cell line A in low 8AA cultures
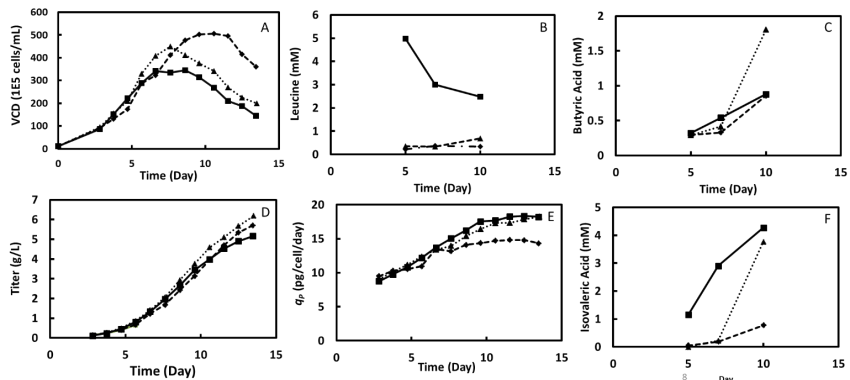
Figure 3: $q_{p}$ enhancing effect of butyrate, isovalerate, and isobutyrate on BCAT1 KO clone of cell line D
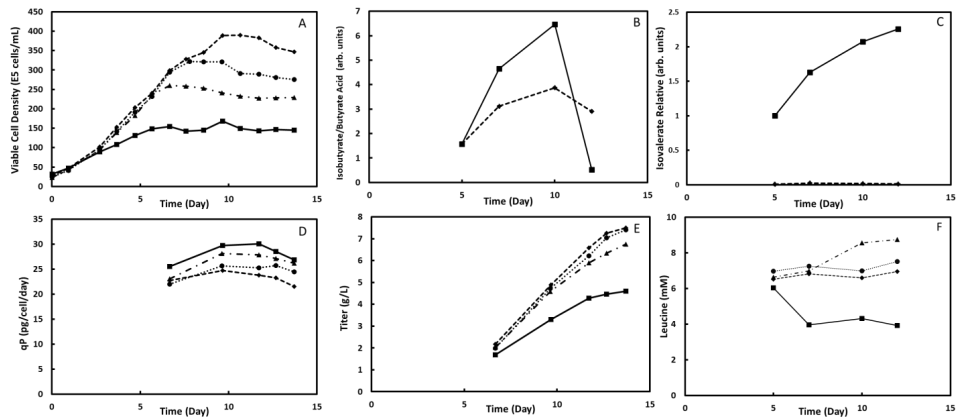
Figure 4: Cell specific production rate of short chain fatty acids inversely linked to lactate production

rate in both cell line $A$ and cell line $D$
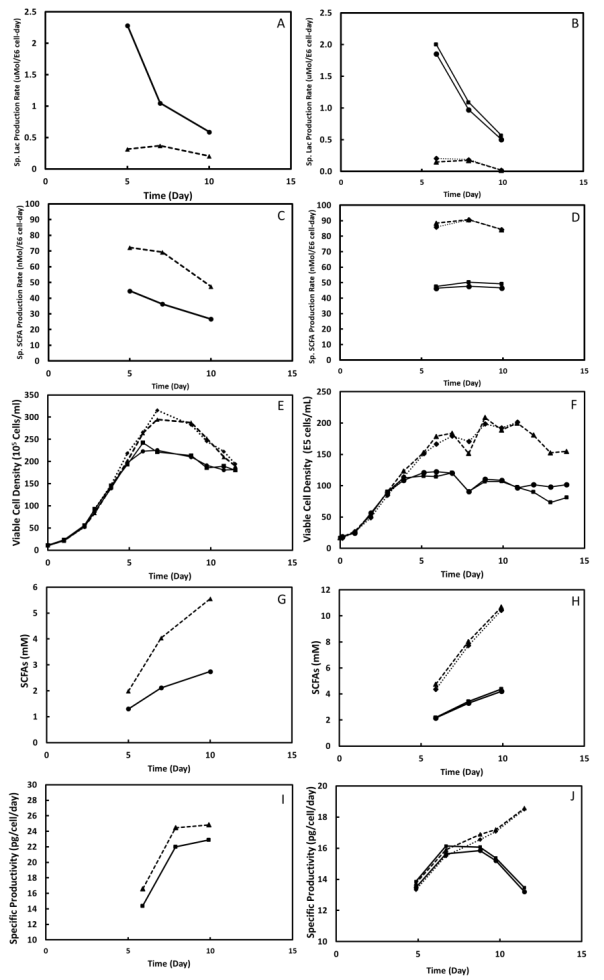
Figure 5: A mechanism linking glycolytic activity to an increase in $q_{p}$
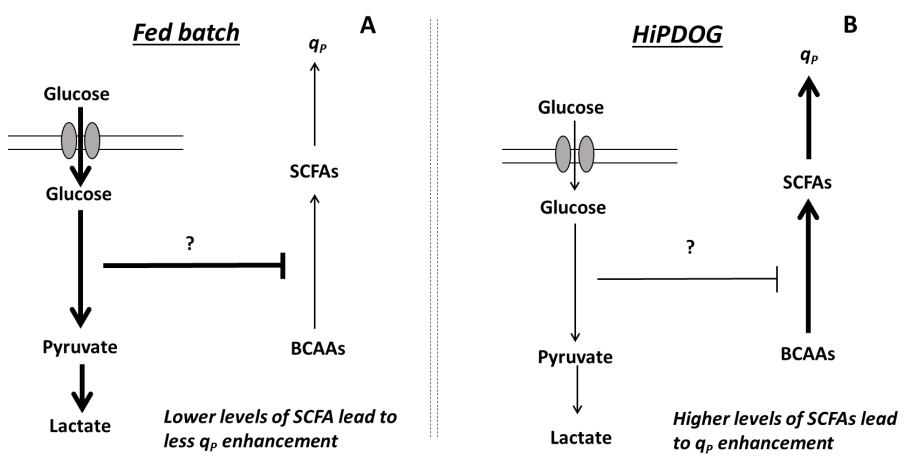
Figure S1: Levels of branched chain amino acids and viable cell densities of cultures in low amino acid

and control conditions from Figure 1
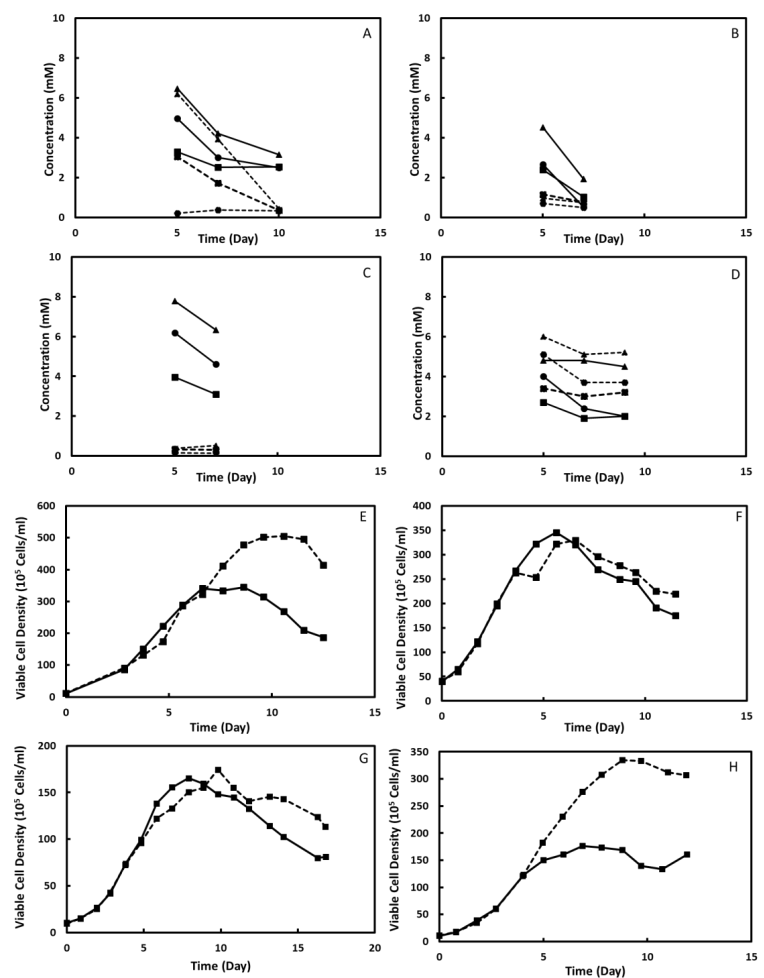
Figure S2: Relative culture milieu accumulations of cholesterol, phosphatidylcholine, ceramides, and lysophospholipids in HiPDOG cultures of WT and BCAT1 KO clone of cell line D
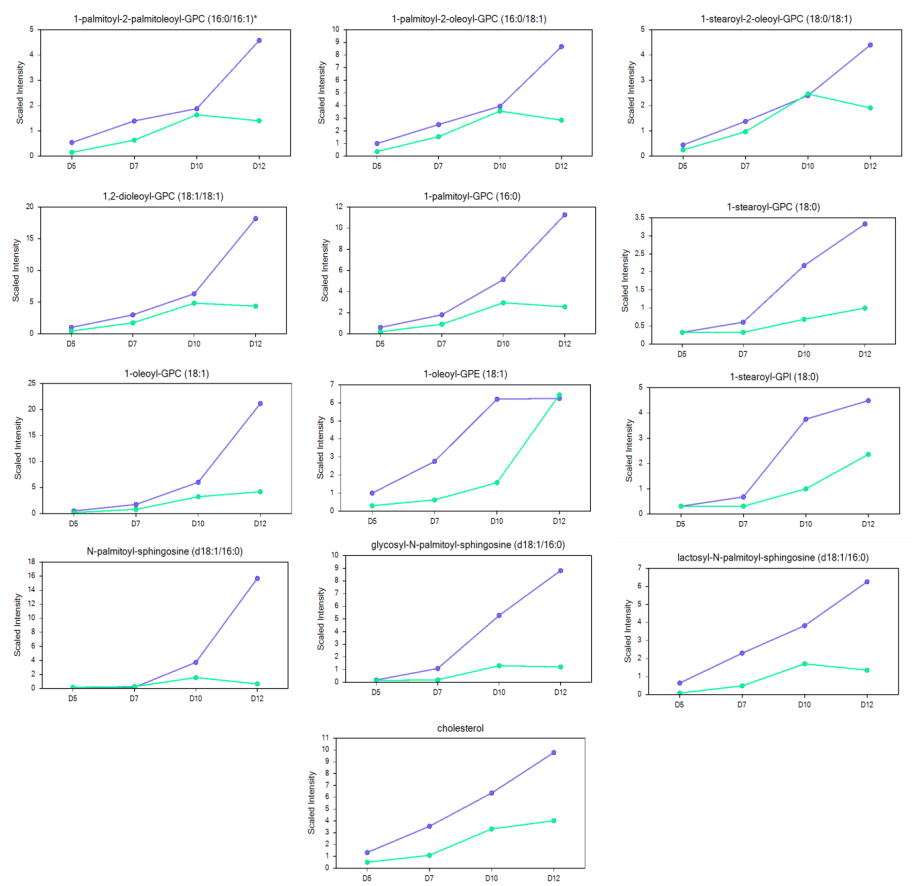
Figure S3: Levels of branched chain amino acids and lysine in cultures of Figure 1 I
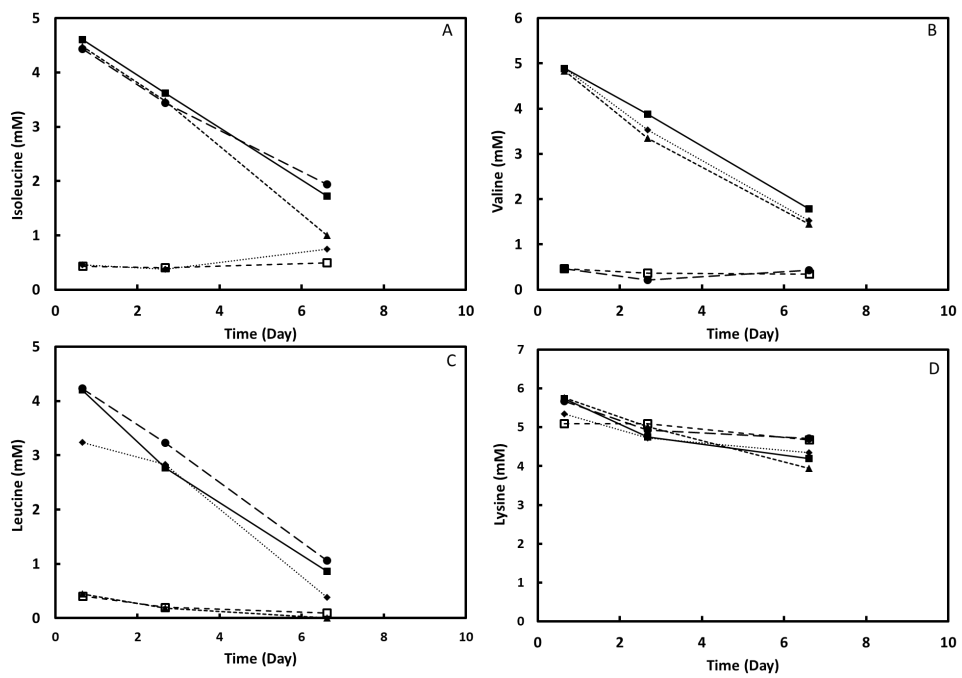

\section{Hosted file}

Figure S4.pdf available at https://authorea.com/users/390259/articles/508402-production-ofbutyrate-and-branched-chain-amino-acid-catabolic-byproducts-by-cho-cells-in-fed-batchculture-enhances-their-specific-productivity 
Figure S5: qP enhancing effect of butyrate, isovalerate and isobutyrate at different concentrations on

cell line B cultivated in low 10AA HiPDOG cultures
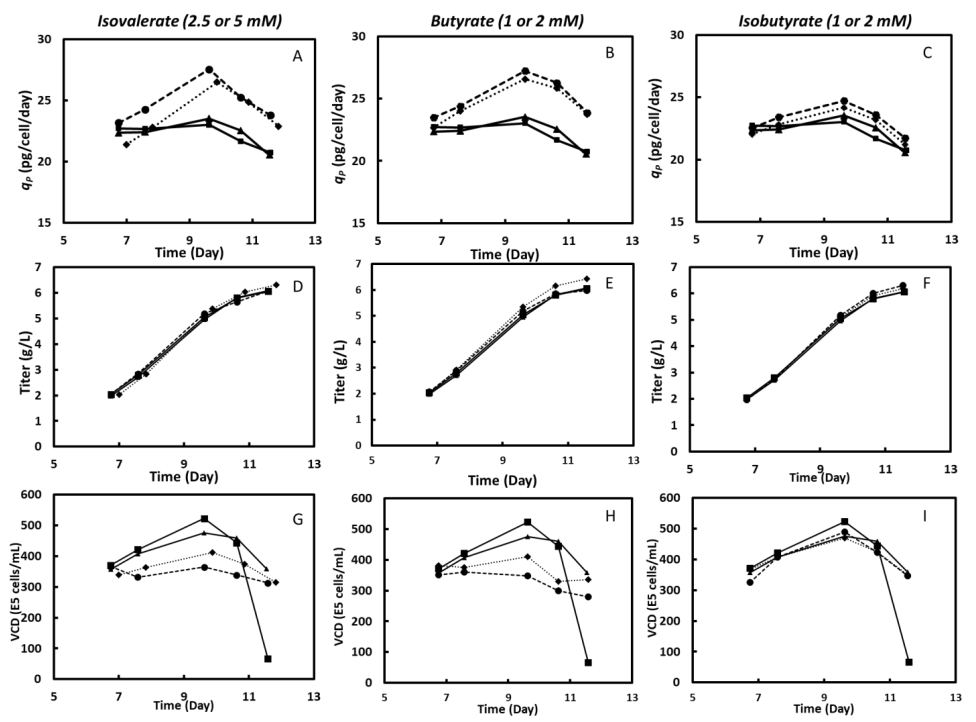
Figure S6: Cell line $\mathrm{G}$ has a higher $q_{p}$ when cultivated in HiPDOG cultures when compared to traditional fed-batch cultures
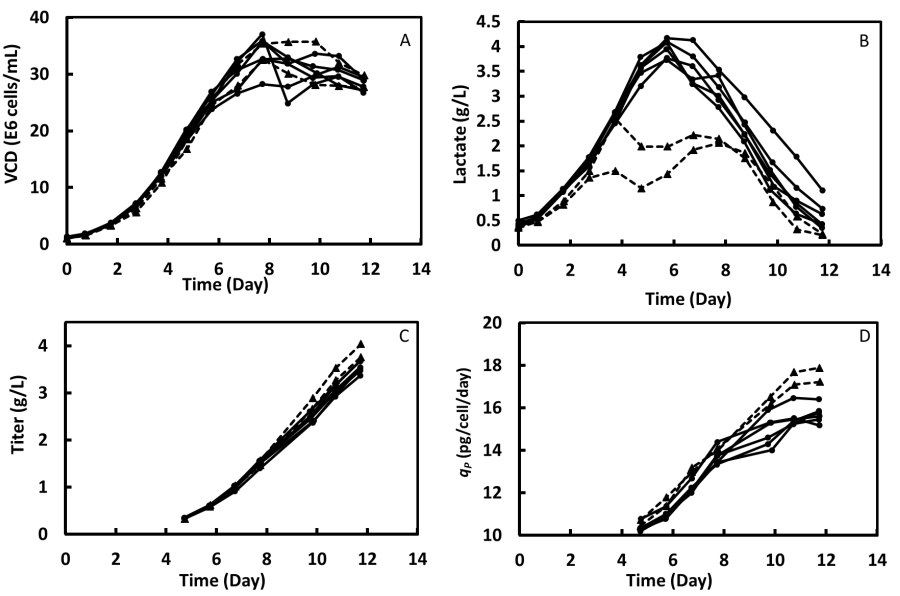
Figure S7: Cell line $\mathrm{H}$, cell line $\mathrm{I}$, cell line $\mathrm{J}$, and cell line $\mathrm{K}$ have increased $q_{\mathrm{p}}$ in HiPDOG cultures when

compared to traditional fed-batch cultures
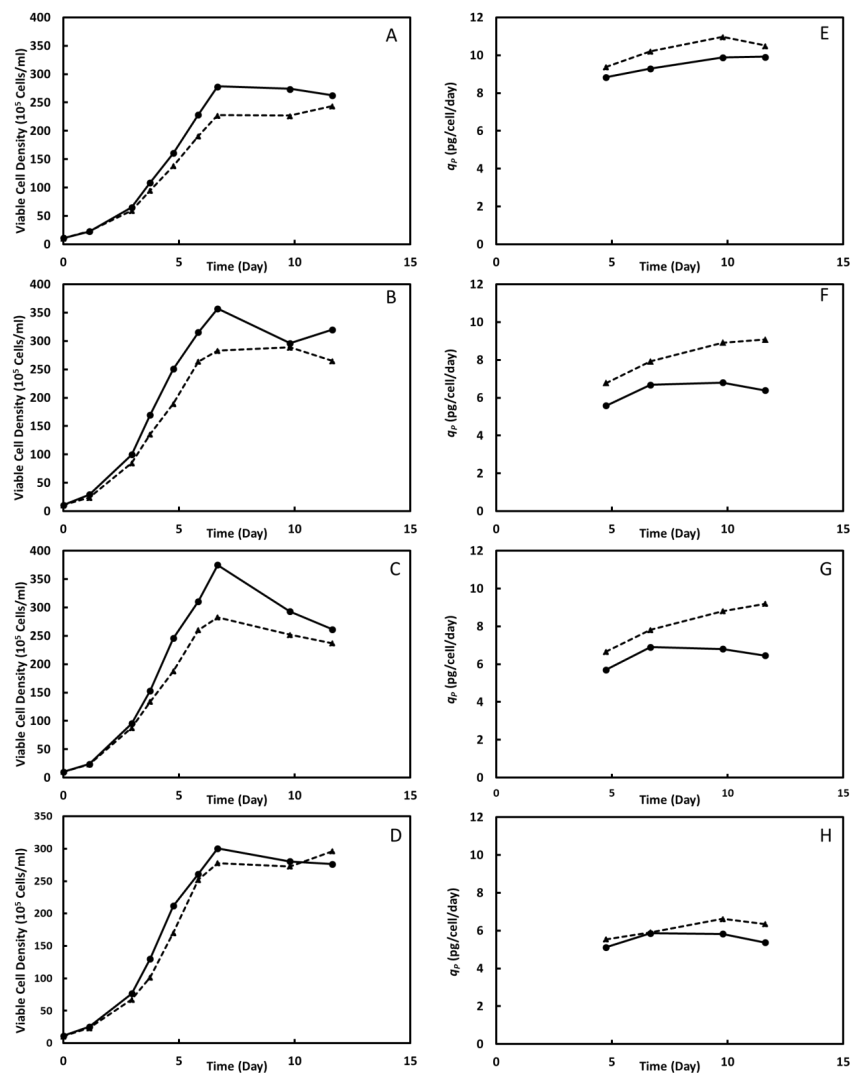